Proyecto de diseño de un condensador con Fuente Eólica para obtención de agua

\title{
Proyecto de diseño de un condensador con Fuente Eólica para obtención de agua
}

\section{Design project for a condenser with Wind Power for water collection}

\section{Projeto de projeto para um condensador com Energia Eólica para coleção de Água}

Otto F. Balseca-Sampedro ${ }^{\mathrm{I}}$

otto.balseca@espoch.edu.ec

Jorge I. Caicedo-Reyes II

isaias.caicedo@espoch.edu.ec

Diego F. Mayorga-Pérez III

d.mayorga@espoch.edu.ec

Javier E. Orna-Chávez ${ }^{\text {IV }}$

javier.orna@espoch.edu.ec

Edwin F. Viteri-Núñez ${ }^{\mathrm{V}}$

eviteri@espoch.edu.ec

Recibido: 21 de agosto de 2017 * Corregido: 18 de octubre de 2017 * Aceptado: 06 de noviembre de 2017

I. Magister en Sistemas de Transporte de Petróleo y Derivados, Ingeniero Mecánico, Docente de la Escuela Politécnica de Chimborazo, Riobamba, Chimborazo, Ecuador.

I. Magister en Diseño Mecánico, Ingeniero Mecánico, Docente de la Escuela Politécnica de Chimborazo, Riobamba, Chimborazo, Ecuador.

III. Magister en Seguridad y Prevención de Riesgos del Trabajo, Ingeniero Mecánico, Docente de la Escuela Politécnica de Chimborazo, Riobamba, Chimborazo, Ecuador.

Iv. Magister en Diseño Producción y Automatización Industrial, Ingeniero Mecánico, Docente de la Escuela Politécnica de Chimborazo, Riobamba, Chimborazo, Ecuador.

v. Magister en Gerencia de Proyectos de Ecoturismo, Ingeniero Mecánico, Docente de la Escuela Politécnica de Chimborazo, Riobamba, Chimborazo, Ecuador. 


\section{Resumen}

Este documento trata la descripción de un proyecto para conseguir un recurso vital, el agua. El agua es el recurso más importante en la vida diaria de la humanidad. Este proyecto intenta compensar la falta de acceso a este recurso mediante condensación de vapor presente en el aire. Se vio la necesidad de hacer este trabajo, dado que, por terremotos, zonas costeras se vieron afectadas, destruyendo edificaciones y tuberías de distribución de este producto vital; lo cual dejó en muy precarias condiciones las zonas afectadas. El objetivo de este trabajo es mostrar cómo condensar agua, limpia y de calidad para el consumo humano, mediante un dispositivo de condensación, para cubrir las necesidades más vitales, que son hidratación diaria, prevención de enfermedades y preparación de alimentos. De esta manera, el diferencial de temperatura entre el aire y el suelo, es el factor clave en el diseño, ya que se da de manera natural sin ningún aporte energético extra. En la historia se han generado distintos mecanismos de condensación, los más eficientes no toman en cuenta la humedad relativa del aire, sino alcanzar el punto de rocío cambiando la temperatura. Este es el mecanismo de funcionamiento del proyecto. Este trabajo fue diseñado para la zona serrana del país, y a partir de este, se puede extrapolar el trabajo para una zona más cálida.

Palabras clave: condensador; punto de rocío; vapor condensado; energías alternativas.

\section{Abstract}

This document deals with the description of a project to obtain a vital resource, water. Water is the most important resource in the daily life of humanity. This project attempts to compensate for the lack of access to this resource by vapor condensation present in the air. The need to do this work was seen, given that, due to earthquakes, coastal areas were affected, destroying buildings and distribution pipes of this vital product; which left the affected areas in very precarious conditions. The objective of this work is to show how to condense water, clean and of quality for human consumption, by means of a condensation device, to cover the most vital needs, which are daily hydration, disease prevention and food preparation. In this way, the temperature differential between the air and the ground is the key factor in the design, since it occurs naturally without any extra energy contribution. In history, different mechanisms of condensation have been generated, the most efficient do not take into account the relative humidity of the air, but reach the dew point by changing the temperature. This is the mechanism of operation of the project. This work was designed for the mountain area of the country and from this, work can be extrapolated to a warmer area. 
Keywords: capacitor; dew point; condensed vapor; alternative energies.

\section{Resumo}

Este documento aborda a descrição de um projeto para obter um recurso vital, a água. A água é o recurso mais importante no cotidiano da humanidade. Este projeto procura compensar a falta de acesso a este recurso por condensação de vapor presente no ar. A necessidade de fazer este trabalho foi vista, dado que, devido a terremotos, as áreas costeiras foram afetadas, destruindo edifícios e tubos de distribuição deste produto vital; que deixou as áreas afetadas em condições muito precárias. O objetivo deste trabalho é mostrar como condensar água, limpar e de qualidade para consumo humano, por meio de um dispositivo de condensação, para cobrir as necessidades mais vitais, que são hidratação diária, prevenção de doenças e preparação de alimentos. Desta forma, o diferencial de temperatura entre o ar eo solo é o fator chave no projeto, pois ocorre naturalmente sem qualquer contribuição de energia extra. Na história, foram gerados diferentes mecanismos de condensação, os mais eficientes não levam em conta a umidade relativa do ar, mas atingem o ponto de orvalho alterando a temperatura. Este é o mecanismo de operação do projeto. Este trabalho foi projetado para a região montanhosa do país, e disso, o trabalho pode ser extrapolado para uma área mais quente.

Palavras chave: capacitor; ponto de orvalho; vapor condensado; energias alternativas.

\section{Introducción}

La escasez de agua potable es la causa principal de enfermedades en el mundo, existiendo hasta 2015, 663 millones de personas sin acceso a agua potable. (Waterseerorg, 2017) (OMS, 2015) En la actualidad, una de cada cinco personas no tiene acceso al agua potable, y cerca de 10 mil personas mueren diariamente por deshidratación y enfermedades causadas por agua no potable (UNICEF, s/f). Este valor aumentará, ya que se prevé que en 2030 el mundo tendrá que enfrentarse a un déficit mundial del 40\% de agua en un escenario climático en que todo sigue igual, debido principalmente a la contaminación y el cambio climático; es de vital importancia cuidar el ambiente también, ya que garantiza recursos limpios para las múltiples actividades diarias y económicas (UNESCO, 2015)

La Tierra contiene unos 525 millones de kilómetros cúbicos de agua. La cantidad de agua que contiene nuestro planeta no ha disminuido ni aumentado en los últimos dos mil millones de años. El 97\% del agua se encuentra en los océanos, y el 2\% permanece congelada. El 80\% del agua que se encuentra 
en los continentes está en la superficie. El 20\% restante se encuentra bajo tierra o en forma de vapor de agua atmosférico. Sólo el 2.5\% del agua que existe en la Tierra es agua dulce. De esa cantidad, el 0.5\% se encuentra en depósitos subterráneos y el 0.01\% en ríos y lagos. Sólo el 0.007\% del agua existente en la Tierra es potable, y esa cantidad se reduce año tras año debido a la contaminación (Fundacionaqua, 2017).

En una absoluta mayoría de casos, las mujeres y niñas son destinadas a la labor de obtener agua, caminando miles de kilómetros e invirtiendo alrededor de 20 horas diarias, tiempo que podría ser utilizado en otras tareas, como cultivo o estudio, que son igualmente de vital importancia (OMS, 2015). Hay que tener en cuenta que esta agua tampoco es potable o tratada (Waterseerorg, 2017).

El Ecuador no es un caso aparte, más de $28 \%$ de la población según el censo de 2010 no tiene acceso a agua por red pública (FCAS, s/f).

Todos los ecuatorianos merecen acceso al agua y que esta sea de calidad, ya que en algunos casos, la población rural, que es la más expuesta a la falta de este recurso, se ve obligada por razones económicas a tomar agua de riego y utilizarla en sus actividades de limpieza. El Gobierno del Buen Vivir ha avanzado con proyectos, y sus obras se ven en la mejora de la calidad de vida de las zonas rurales; sin embargo, realizar la red de agua potable a todos los rincones del Ecuador se hace difícil y, por ende, muy costoso. Estos proyectos se valoran en ciento de millones de dólares y se tiene previsto su culminación cerca del 2030 (Gobierno de Ecuador, 2016).

La cobertura de agua en la provincia de Chimborazo es del 42,4\% (Gobierno de Ecuador, 2016).

El Estado busca cumplir con los derechos humanos, que la población obtenga acceso a una vida digna y con acceso a todos los recursos necesarios para su bienestar (art. 25, Derechos Humanos) (Asamblea Nacional, s/f).

La recuperación y transformación del Estado es el pilar político de la Revolución Ciudadana. Para obtener la democracia y caminar hacia el Socialismo del Buen Vivir; se han transformado las instituciones públicas para ponerlas al servicio ciudadano; se ha alcanzado la apropiación de recursos por parte del Estado y la recuperación de su autonomía relativa; y se ha fomentado la apertura de mecanismos y procesos de participación ciudadana (Secretaría Nacional de Planificación y Desarrollo, 2013). 
Este es el objetivo 1 presente en el Buen Vivir del Gobierno Ecuatoriano; recuperar los recursos del Estado y obtener mayor participación ciudadana. Se busca la construcción de más democracia en su doble vertiente, esto es la consolidación de institucionalidad estatal pero también social, seguir construyendo y dando significado a lo público, a lo común, a lo comunitario.

El objetivo de este trabajo es mostrar cómo condensar agua, limpia y de calidad para el consumo humano, mediante un condensador, que es un futuro proyecto en comunidades, en conjuntos poblacionales sin acceso permanente al recurso humano vital que es el agua.

El condensador de agua o pozo aéreo, es un proyecto que existe desde hace cientos de años, el primero encontrado funcional, es el condensador de Zibold (Nikolayev, y otros, 1996) ubicado sus restos en la antigua ciudad de Fedosia, Ucrania. Estos restos datan del siglo VI, reconstruido por F. I. Zibold en 1912, y se basan en la captación de rocío presente en cualquier zona del mundo húmeda o árida con cualquier tipo de temperatura, al contrario que la niebla que se forma con $100 \%$ de humedad relativa en pocas zonas propicias. Actualmente, se ha mejorado este proyecto, basado en las distintas ubicaciones donde se encuentra y se ha generado diferentes modelos, el más reciente, el proyecto WaterSeer (Waterseerorg, 2017), capaz de producir 42 litros diarios ocupando menos de un metro cuadrado de superficie, incluso existen fundaciones para lograr obtener agua como OPUR (Organización Por la Utilización del Rocío, 2013), que promueven el uso y obtención de agua mediante el rocío.

Teniendo en cuenta que la condensación es un medio económico y limpio de obtener agua, es una idea verdaderamente atractiva; facilita el acceso al recurso, es un agua más limpia que la de lluvia o incluso que la que pasa por tuberías (Muselli, y otros, 2002) (Bautista-Olivas, y otros, 2011), no necesita de una fuente energética a base combustibles fósiles o eléctrica y, simplemente, necesita un cálculo termodinámico preciso para ser eficiente. Para la zona de trabajo se tienen datos promedio de: temperatura ambiente $\left(13^{\circ} \mathrm{C}\right)$, temperatura de suelo $\left(22^{\circ} \mathrm{C}\right)$, Humedad relativa $(62.6 \%)$ Velocidad del viento $(2.6 \mathrm{~m} / \mathrm{s})$ y Presión atmosférica $(73.087 \mathrm{kPa})$. 


\section{Desarrollo}

El hogar promedio ecuatoriano, consta de 3.8 miembros según datos del Inec (INEC, 2016). Una persona consume en promedio 150 litros del agua al día. Es decir, que esto equivaldría a 570 litros de agua diaria en una familia ecuatoriana. (CEPAL, 2017)

De estos datos, según la OMS; se hace necesario resaltar que el consumo de agua necesario es de 2.9 litros en hombres adultos, 2.2 litros de agua en mujeres adultas y de 1 litro en niños. Extrapolando esto a una familia de 4 miembros, serían 7.1 litros de consumo directo. (OMS, 2003)

Para cocinar, el promedio por hogar es de 1.2 a 2 litros por comida diaria; lo que vendría a dar 10 litros diarios en la preparación de comidas.

En lo que respecta a higiene, el agua potable es muy importante, ya que lavarse las manos previene muchas enfermedades y sobretodo la disentería, principal causa de muerte en los países sin acceso al agua potable. El consumo de agua diario por persona en lo que respecta a lavarse las manos es de 2 litros, lo que conlleva a un gasto de 8 litros en un hogar de 4 personas.

Suponiendo con estos datos de la OMS, que del total de agua consumida en un hogar de este país, alrededor de 25 litros son vitales diariamente. Con este proyecto, se enfocará en obtener los cálculos para condensar como mínimo, un volumen de 25 litros diarios, y con un volumen óptimo de 40 litros por día. (OMS, 2015)

Si analizamos los datos de temperatura media del suelo y atmosférica en Riobamba, obtendremos que el suelo esté con una temperatura superior a la del aire; sin embargo, este fenómeno no ocurre siempre a lo largo del día; mas sí la mayor parte del día. 


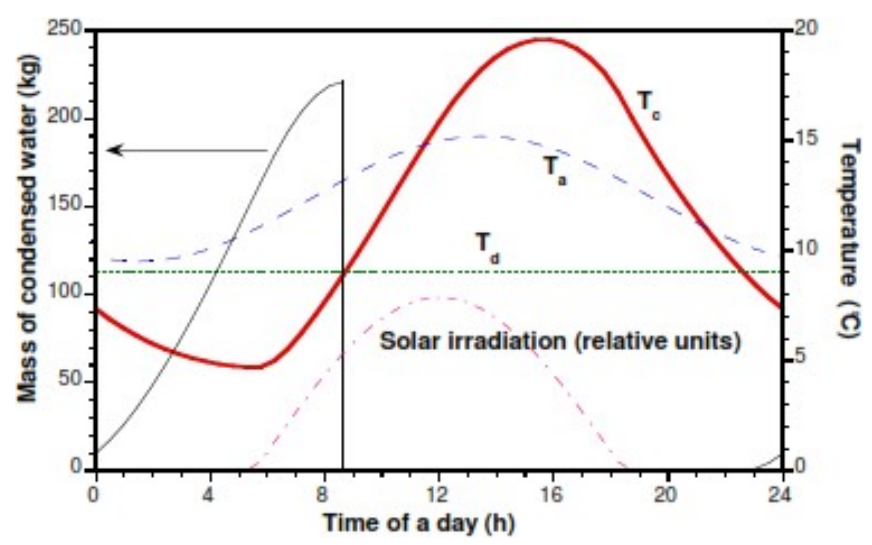

Fig. 1 Evolución de temperaturas a lo largo del día en el condensador Zibold y su aire circundante [8]. La línea verde (Td) representa la temperatura de condensación, la línea azul segmentada (Ta) es la temperatura del aire circundante, la línea azul continua es la masa de agua condensada, la línea roja (Tc) simboliza la temperatura del condensador.

Si se observa la figura 1 con detenimiento, y observamos el eje de la $\mathrm{X}$, veremos que éste, representa las horas del día. Y que la condensación de agua es de aproximadamente 10 horas, lo que quiere decir que durante 14 horas, la temperatura es demasiado alta para condensar y que se repite el fenómeno analizado al estudiar las temperaturas promedio: el condensador (suelo en nuestro caso de estudio) posee una temperatura mucho más elevada.

Para desechar este problema de eficiencia, se analizar la temperatura a la que debe estar el aire en función de la temperatura del suelo $\left(22^{\circ} \mathrm{C}\right)$, la cual será convertida en temperatura de saturación.

Se analizará el fenómeno como un enfriamiento de aire con condensación. Para el análisis, y su simplificación se utilizará un esquema simple (fig. 2), pero en realidad el aire debe ingresar y salir por el mismo agujero de ventilación.

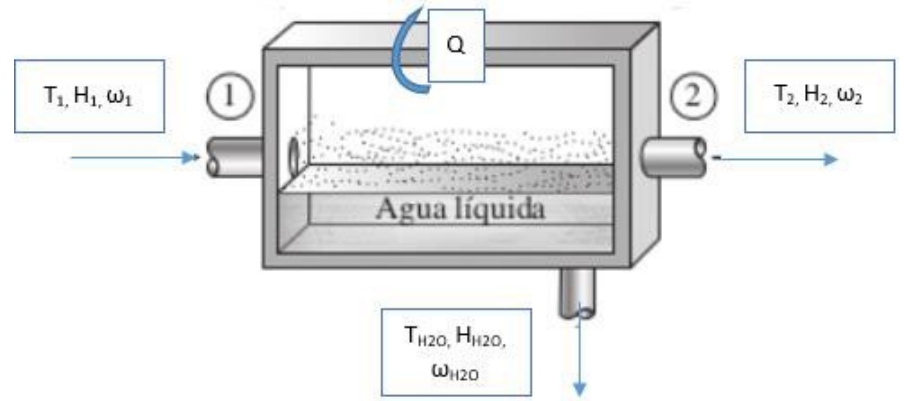


Fig. 2 Proceso de enfriamiento con condensación. Tomado de: ÇENGEL, Y. A.; BOLES, M. A.: Termodinámica. Ed. McGraw-Hill: México, D.F., 2009. (Sexta edición en Español correspondiente a la sexta edición original en inglés). ISBN 978-9701072868.

Se debe tener en cuenta que la variación de temperatura se realiza desde el ingreso, donde se encontrará la temperatura de inicio del aire con el vapor, que será la temperatura ambiente; de la misma manera, tendrá un enfriamiento hasta una temperatura bajo el suelo, la temperatura final, cuyo valor óptimo será la temperatura de condensación.

Para analizar la temperatura óptima del aire, poniendo la temperatura del suelo como temperatura de rocío, y teniendo datos de presión atmosférica de Riobamba y humedad relativa en este sitio, con los datos tomados del INHAMI ubicado en la politécnica; se ingresa al programa CYTSoft Psychrometric Chart 2.2, el cual indica los valores que se muestran en la tabla 1:

\begin{tabular}{|c|c|c|}
\hline $\mathrm{DP}$ & $\checkmark$ & 22 \\
\hline $\mathrm{RH}$ & $\checkmark$ & 62.06 \\
\hline DB: & & 30.05843343 \\
\hline WB: & & 23.73690902 \\
\hline RH: & & 62.06000000 \\
\hline W: & & 23.43113151 \\
\hline h: & & 90.17525156 \\
\hline v: & & 1.23536446 \\
\hline DP: & & 22.00000000 \\
\hline d: & & 0.80947772 \\
\hline dm: & & 0.82844470 \\
\hline AW: & & 0.01896698 \\
\hline vp: & & 2653.37316783 \\
\hline
\end{tabular}

Tabla 1 Datos arrojados por el programa CYTSoft Psychrometric Chart 2.2.

De esta manera, la temperatura de ingreso del aire debe ser de: $30.1^{\circ} \mathrm{C}$, que viene indicado por Dry Bulb o Bulbo Seco.

Conservación de masas

$$
\dot{m} \dot{m}_{1}=\dot{m}_{2}=\dot{m}_{a}+m_{\dot{H}_{2} O}
$$


Se sabe que se debe conseguir un flujo volumétrico de $V_{H_{2}}{ }_{O}$ de 40 litros por día, por lo que transformando se obtiene: $m_{\mathrm{H}_{2} \mathrm{O}}^{\cdot}=4.62962963 \times 10^{-7} \frac{\mathrm{m}}{\mathrm{s}}^{3} * \delta_{\mathrm{H}_{2} \mathrm{O}_{T_{2}}}$. El valor de densidad, al ser un líquido, se puede obtener de tablas con el dato de la temperatura de suelo o $\mathrm{T}_{\mathrm{H} 2 \mathrm{O}} \underset{v}{1}$ que es el volumen específico. Lo que nos arroja un dato continuo, ya que no se obtendrá en determinados lapsos de tiempo cuando las condiciones sean idóneas, sino que por mano humana, las condiciones siempre serán idóneas.

También, $\underset{1}{m}=\delta * v * A \perp$, donde v es la velocidad de aproximadamente $2,6^{m}, A \perp \underline{s}$ es el área perpendicular de ingreso que podría ser de $0,5 \mathrm{~m}^{2}$ y esto se iguala a: $A \perp=\pi * r^{2}$ y r sería de $0,4 \mathrm{~m}, \mathrm{y}$ $\delta$ es la densidad que va en función de la temperatura utilizando la ecuación de gases ideales: $\delta=\frac{P}{R T}$, en Riobamba la presión es de 73087,32 Pa y la $\mathrm{R}$ es la constante de gas del aire $287 \frac{\mathrm{l}}{\mathrm{kg} * K}$. Donde quedaría: $m_{1}=595,83 \frac{K g * K}{s} * \frac{1}{T_{1}}$

Para obtener unos valores lo más ajustados posibles, debemos tener en cuenta que todos los factores varían con la temperatura.

En el caso de estudios de temperatura, se debe hallar una ecuación que defina la temperatura del aire atmosférico según el tiempo transcurrido del día. De la misma manera, debe hacerse con la temperatura del suelo, y de esta manera hallar la temperatura de saturación.

También se hace necesario, conocer la profundidad del Condensador, para tener una temperatura más o menos uniforme, con la que trabajar, y la que será temperatura del suelo y, posiblemente, la de saturación. Se puede calcular la temperatura del suelo suponiendo un suelo homogéneo de transmisibilidad constante, a una determinada hora del día con la siguiente ecuación:

$T(z, t)=T_{m}-A_{s} e^{-z \sqrt{\frac{\pi}{365 \alpha}}} \cos \left[\frac{2 \pi}{365}\left(t-t_{0}-\frac{z}{2} \frac{\bar{\pi}}{365 \alpha}\right)\right](6)$

Donde:

$T(z, t)$ es la temperatura en ${ }^{\circ} \mathrm{C}$ del suelo en el tiempo t en días a una profundidad $\mathrm{z}$. 
$T_{m}$ es la temperatura media anual del suelo en ${ }^{\circ} \mathrm{C}$ a una profundidad donde no se aprecien las variaciones de temperatura.

$A_{s}$ es la oscilación de la temperatura superficial.

$t_{0}$ es el desfase de días.

$\alpha$ es la difusividad témica del suelo en $\frac{m^{2}}{d i ́ a}$

Otra forma de determinación de la temperatura del suelo es basado en la recolección de datos experimentalmente (INSTITUTO PARA LA DIVERSIFICACIÓN Y AHORRO DE LA ENERGÍA, 2010), pero requeriría de la ubicación de termocuplas enterradas a una distancia de 2 metros, donde disminuiría la temperatura de saturación o suelo en este caso, haciendo que también descienda la temperatura de aire atmosférica requerida.

En el momento del modelado, hay que tener límites, ya que si la temperatura del suelo, no llega a ser como mínimo la temperatura de saturación $\left(T_{\mathrm{H}_{2} \mathrm{O}} \leq T_{\text {sat }}\right)$, entonces el condensado nunca se producirá, como ocurre con la mayoría de zonas del mundo durante las horas más perpendiculares de radiación solar, pero para esto se genera el mecanismo de aumento de temperatura, que aprovechando esta radiación, podría hacerse con un invernadero sobre el condensador que establezca la temperatura del aire.

Una vez realizados los cálculos, se obtienen los siguientes resultados de diseño. En la tabla 2, se incluyen los datos de ingreso.

Tabla 2 Datos de Diseño del Condensador.

\begin{tabular}{|c|c|c|}
\hline & Variable & Valor Numérico \\
\hline & T Saturación & $22^{\circ} \mathrm{C}$ \\
\hline & P atm Riobamba & $73.078 \mathrm{kPa}$ \\
\hline & Humedad Relativa & $62.60 \%$ \\
\hline & T Aire (Ideal) & $30.1^{\circ} \mathrm{C}$ \\
\hline & Velocidad Ingreso & $2.6 \mathrm{~m} / \mathrm{s}$ \\
\hline & Velocidad Condensador & $18.5 \mathrm{~m} / \mathrm{s}$ \\
\hline 394 & $\begin{array}{l}\text { Vol. 4, núm. 1, enero, 2018, pp } \\
\text { Otto F. Balseca-Sampedro, Jo } \\
\text { Viteri-Núñez }\end{array}$ & yorga-1 \\
\hline
\end{tabular}


Proyecto de diseño de un condensador con Fuente Eólica para obtención de agua

\begin{tabular}{|l|l|}
\hline Rad. Entrada & $0.40 \mathrm{~m}$ \\
\hline Rad. Cilindro & $0.15 \mathrm{~m}$ \\
\hline Rad. Esfer & $0.221 \mathrm{~m}$ \\
\hline Volumen Condensado & $0.04 \mathrm{~m} 3$ \\
\hline
\end{tabular}

\section{Conclusiones}

La calidad del agua obtenida por captación de rocío o condensación genera un agua de alta calidad y pureza, esto hace que el agua potable pueda ser accesibles en zonas remotas; y podría ser la solución a la escasez de este recurso en zonas rurales o pobres, en todo el mundo; sobre todo en lugares donde este recurso no pueda llegar en tuberías de red pública, o éstas se hallar roto por algún desastre natural.

Los condensadores actuales, debido a la radiación solar y la tendencia del suelo a retener el calor, tienen horas de trabajo menores a las de pausa debido a las condiciones climáticas. Si a esto se le suma las condiciones de las zonas no favorables (Tsuelo>Taire), se obtiene un bajo rendimiento, que podría aumentarse haciendo que el aire de ingreso se encuentre a la temperatura óptima para conseguir un condensado a lo largo del día, lo cual equivale a reducir las dimensiones del condensador, y no tener escasez de agua en las horas de no condensado; esto lleva a un ahorro de dinero y evitar la necesidad de ir a recolectar agua en ríos y demás fuentes.

Este condicionamiento de aire puede solucionarse con una implementación extra de un invernadero que eleve la temperatura aprovechando la radiación solar que perjudica a los condensadores; lo que lleva a no usar una fuente de energía extra.

Se concluye que las condiciones ideales de condensación para implementar este dispositivo son en zonas con temperatura ambiental cercana a $20^{\circ} \mathrm{C}$ y humedad aproximada de $70 \%$, con ello se garantiza y eficiente funcionamiento del dispositivo según se muestra en los cálculos.

Para la extracción del agua condensada del interior de la cámara se la puede realizar con la instalación de bomba de succión manual, lo cual evitaría la contaminación del líquido por manipulación del mismo. 
El Gobierno de Buen Vivir, quiere garantizar el acceso a este recurso vital, sin embargo, se ven expectativas de culminación de costosos proyectos de cientos de millones de dólares cerca del 2030, pero mientras tanto, este proyecto de Condensador podría suplir esta necesidad en zonas remotas.

\section{Referencias bibliográficas}

Asamblea Nacional. s/f. Constitución del Ecuador. [En línea] s/f. [Citado el: 05 de mayo de 2017.] http://www.asambleanacional.gov.ec/documentos/constitucion_de_bolsillo.pdf.

BAUTISTA-OLIVAS, Ana L., y otros. 2011. La humedad atmosférica como fuente opcional de agua para uso doméstico. Agrociencia 45 (3). 2011.

CEPAL. 2017. Diagnóstico de las estadísticas del agua en Ecuador. [En línea] 2017. [Citado el: 02 de febrero de http://aplicaciones.senagua.gob.ec/servicios/descargas/archivos/download/Diagnostico\%20de\%20la s\%20Estadisticas\%20del\%20Agua\%20Producto\%20IIIc\%202012-2.pdf.

FCAS. s/f. Ecuador. [En línea] s/f. [Citado el: 02 de febrero de 2017.] http://www.fondodelagua.aecid.es/es/fcas/donde-trabaja/paises/ecuador.html.

Fundacionaqua. 2017. Principales datos del agua en el mundo. [En línea] 2017. [Citado el: 02 de febrero de 2017.] https://www.fundacionaquae.org/wiki-aquae/datos-del-agua/principales-datos-delagua-en-el-mundo/.

Gobierno de Ecuador. 2016. EStrategia Nacional de agua potable y saneamiento. [En línea] 2016. [Citado el: 13 de febrero de 2017.] http:/www.agua.gob.ec/wpcontent/uploads/downloads/2016/07/REVISTA-SENAGUA.compressed.pdf.

INEC. 2016. INEC actualiza año base del IPP. [En línea] 2016. [Citado el: 05 de febrero de 2017.] http://www.ecuadorencifras.gob.ec/inec-actualiza-ano-base-delipp/?option=com_content\&view=article $\&$ id=422\%3Ase-reduce-el-tamano-de-los-hogaresecuatorianos \&catid $=68 \% 3$ Aboletines $\&$ Itemid $=51 \&$ lang $=$ es.

INSTITUTO PARA LA DIVERSIFICACIÓN Y AHORRO DE LA ENERGÍA. 2010. Guía técnica de diseño de sistemas de bomba de calor geotérmica. Madrid: IDAE, 2010. 
Muselli, M., y otros. 2002. Dew water collector for potable water in Ajaccio (Corsica Island, France). Atmospheric Research, 64(1). 2002.

Nikolayev, V.S., y otros. 1996. Water recovery from dew. 1996.

OMS. 2003. Domestic Water Quantity,. [En línea] 2003. [Citado el: 03 de febrero de 2017.] http://www.who.int/water_sanitation_health/diseases/WSH03.02.pdf?ua=1.

-. 2015. Informe 2015 del PCM sobre el acceso a agua potable y saneamiento: datos esenciales. [En línea] 2015. [Citado el: 02 de http://www.who.int/water_sanitation_health/monitoring/jmp-2015-key-facts/es/.

Organización Por la Utilización del Rocío. 2013. ¿Por qué OPUR? [En línea] 2013. [Citado el: 02 de febrero de 2017.] http://www.opur.fr/esp/question2_esp.htm.

Secretaría Nacional de Planificación y Desarrollo. 2013. El Socialismo del buen vivir. [En línea] 2013. [Citado el: 02 de diciembre de 2016.] http://www.buenvivir.gob.ec/69.

UNESCO. 2015. Informe de las Naciones Unidas sobre los recursos hídricos en el mundo 2015. [En línea] 2015. [Citado el: 15 de febrero de 2017.$]$ http://www.unesco.org/new/fileadmin/MULTIMEDIA/HQ/SC/images/WWDR2015Facts_Figures_ SPA_web.pdf.

UNICEF. s/f. La infancia y el agua: estadísticas generales. [En línea] s/f. [Citado el: 02 de marzo de 2017.] https://www.unicef.org/spanish/wash/index_31600.html.

Waterseerorg. 2017. The Perfect Answer. [En línea] 2017. [Citado el: 20 de marzo de 2017.] https://www.waterseer.org/. 